\title{
Associative aspects of conditioned analgesia evoked by a discrete CS
}

\author{
ROBERT T. ROSS and ALAN RANDICH \\ University of Iowa, Iowa City, lowa
}

\begin{abstract}
Four experiments with rat subjects assessed conditioned analgesia evoked by a discrete visual CS repeatedly paired with a shock US. In Experiment 1, rats that received CS-US pairings followed by a hot-plate test of pain sensitivity in the presence of the CS showed significantly longer response latencies than groups of rats receiving a variety of Pavlovian control procedures, including CS and US explicitly unpaired, CS-alone, US-alone, and apparatus-alone. Experiment 2 showed that two CS-US pairings were required for the CS to evoke conditioned analgesia and that additional CS-US pairings did not augment the observed analgesia. Experiment 3 demonstrated extinction of conditioned analgesia in groups that received CS-alone presentations. Experiment 4 found that conditioned analgesia established with the present procedures was unaffected by prior administration of the opiate receptor antagonist naloxone. Experiments 1 and 3 also demonstrated a number of situations in which freezing behavior (used to index conditioned fear) could be dissociated from hot-plate response latencies (used to index conditioned analgesia). These results are discussed in terms of how associative learning processes mediate acquisition of conditioned analgesia.
\end{abstract}

Simple exposure to one of a variety of noxious stimuli can result in a temporary decrease in an organism's sensitivity or reactivity to painful stimulation-a phenomenon referred to as stress-induced analgesia because this alteration in the normal response to noxious stimuli is assumed to be mediated by endogenous systems capable of inhibiting the transmission or processing of information about pain (Amir, Brown, \& Amit, 1980; Bodnar, Kelly, Brutus, \& Glusman, 1980; Bolles \& Fanselow, 1982; Ross \& Randich, 1984; Spiaggia, Bodnar, Kelly, \& Glusman, 1979). Similarly, a number of studies have demonstrated that originally neutral stimuli that occur in temporal and/or spatial proximity to a noxious stimulus can also acquire the capacity to evoke analgesia (Chance, 1980; Drugan, Moye, \& Maier, 1982; Fanselow \& Baackes, 1982; Hayes, Bennett, Newlon, \& Mayer, 1978; Watkins, Cobelli, \& Mayer, 1982), although perhaps not necessarily by utilizing the same endogenous substrate activated by the noxious stimulus itself (Watkins \& Mayer, 1982).

The primary account of the processes underlying acquired or conditioned analgesia is one emphasizing a critical role for associative learning (Bolles \& Fanselow, 1980; Chance, 1980; Riley, Zellner, \& Duncan, 1980; Watkins et al., 1982). In general, an associative analysis

This research was supported by National Institutes of Health Grant NS18241 to A. Randich. R. T. Ross was supported by Postdoctoral Training Fellowship MH15773 from the National Institutes of Health and by a Killam postdoctoral fellowship from Dalhousie University. The naloxone was a generous gift from the National Institute of Drug Abuse. We thank Vincent M. LoLordo for his many useful comments regarding this manuscript. Requests for reprints should be addressed to Robert T. Ross, Department of Psychology. Dalhousie University. Halifax. NS. Canada B3H 4JI. views analgesia elicited by presentation of a stimulus previously paired with the occurrence of the noxious stimulus as a conditioned response (CR) established according to the laws of Pavlovian conditioning. Sufficient evidence is now available to support the viability of an associative interpretation of conditioned analgesia for situations in which the conditioned stimulus (CS) is provided by the experimental apparatus cues (or contextual stimuli) and the unconditioned stimulus (US) is electric shock. For example, (1) acquisition of conditioned analgesia has been demonstrated in groups of rats that receive paired CS-US presentations, but not for groups of rats that receive a variety of Pavlovian control procedures (Fanselow \& Baackes, 1982; MacLennan, Jackson, \& Maier, 1980); (2) basic acquisition functions have been delineated for a number of conditioning preparations (Chance, 1980; Chance \& Rosecrans, 1979a, 1979b; Chance, White, Krynock, \& Rosecrans, 1979; Drugan et al., 1982; Fanselow \& Baackes, 1982); and (3) extinction of conditioned analgesia following repeated or prolonged exposure to the CS alone has been reported (Drugan et al., 1982; Fanselow, 1984).

The availability of evidence on conditioned analgesia in situations in which the CS is a discrete event, rather than provided by diffuse contextual stimuli, is less extensive. This is somewhat surprising, given the potential importance of conditioned analgesia for the analysis of aversive learning and behavior (e.g., Riley et al., 1980; Ross \& Randich, 1984), in which studies typically employ either a discrete auditory or visual stimulus as a CS. However, Fanselow and Bolles (1979) have shown acquisition of conditioned analgesia to an auditory CS in rats receiving forward (i.e., CS-US) pairings of a tone and shock, but not in rats receiving backward (i.e., US-CS) 
pairings of the same stimuli. Watkins et al. (1982) replicated this effect and provided additional controls for nonassociative effects such as pseudoconditioning and sensitization. Watkins et al. also reported extinction of conditioned analgesia in rats that received nonreinforced presentations of the tone CS following conditioning. Oliverio and Castellano (1982) found acquisition of conditioned analgesia in mice that received either simultaneous presentations of a tone CS and a shock US or contextual stimuli-US pairings, but not in mice that received only nonreinforced presentations of either CS. Oliverio and Castellano further determined that 60 nonreinforced presentations of the tone CS were ineffective in extinguishing conditioned analgesia. Although there is no reason to question the basic outcomes of these studies, there are some difficulties with interpretation regarding the associative analysis of conditioned analgesia. For example, Fanselow and Bolles (1979) did not include a direct assessment of pain sensitivity; analgesia was inferred from the occurrence of one of the rat's species-specific defense behaviors (i.e., freezing) and the reversal of this response by naloxone pretreatment. Oliverio and Castellano (1982) and Watkins et al. (1982) reported contradictory findings regarding the effectiveness of CS-alone presentations in extinguishing conditioned analgesia, but neither study employed an appropriate control procedure for assessing this effect. Thus, there was clearly a need for additional data with which to evaluate the associative account of conditioned analgesia for situations in which the CS was a discrete stimulus.

To meet this need, we undertook the following experiments, in which conditioned analgesia was evaluated in rats that were given pairings of a discrete visual CS and a shock US. In these experiments, a light CS and a shock US were presented to a rat in an apparatus consisting of a copper plate surface enclosed by a clear Plexiglas cylinder. Assessment of analgesia was conducted in an identical apparatus in which the copper plate surface was thermostatically controlled; this was a hot-plate assay of pain sensitivity. Two identical apparatuses were used for conditioning and assessment of analgesia to minimize the possibility of a generalization decrement between conditioning and testing situations. In a preceding set of experiments (Ross \& Randich, 1984), this experimental apparatus had been used to investigate unconditioned analgesia evoked by the shock US that was used in the present studies. Specifically, unconditioned analgesia in these studies (1) depended upon the use of a relatively intense shock ( $2 \mathrm{~mA})$, (2) systematically increased in magnitude as shock duration increased (range $0.5-2.0 \mathrm{sec}$ ), (3) was of short duration (approximately $30 \mathrm{sec}$ ), and (4) was not attenuated by prior administration of the opiate receptor antagonist naloxone. These outcomes set the stage for the following series of experiments on conditioned analgesia. Experiment 1 compared conditioned analgesia evoked by a visual CS in a CS-US paired condition with various control groups that included CS and US explicitly unpaired, CS alone, US alone, and appara- tus alone. Experiment 2 varied the number of CS-US pairings to determine the conditions sufficient for acquisition of analgesia. Experiment 3 assessed the conditions necessary for the extinction of conditioned analgesia; it included controls for the simple passage of time. Experiment 4 was a preliminary examination of the efficacy of the opiate receptor antagonist naloxone in reversing conditioned analgesia evoked by a visual CS. Finally, in all studies, an index of conditioned fear (freezing) was recorded in the presence of the visual CS during conditioning.

\section{EXPERIMENT 1}

Experiment 1 compared acquisition of conditioned analgesia in a group of rats that received paired presentations of a visual CS and a shock US with that in control groups that received exposure to the CS and US explicitly unpaired, US only, CS only, or apparatus only. The purpose of this experiment was to establish, by systematically assessing nonassociative contributions to the behavior, the associative basis of conditioned analgesia elicited by the visual CS. In addition, since a number of studies had indicated that contextual stimuli paired with a shock US might acquire the capacity to evoke analgesia (Chance, 1980; Drugan et al., 1982; Fanselow \& Baackes, 1982; Hayes et al., 1978) and the US-only treatment of the present experiment might be expected to condition contextual stimuli, a context testing procedure was also included to determine the relative contributions of the visual $\mathrm{CS}$ and contextual stimuli to the behavior.

\section{Method}

Subjects. The subjects were 80 experimentally naive male Sprague-Dawley rats obtained from Hormone Assay Laboratories in Chicago. The rats weighed approximately $225 \mathrm{~g}$ and were 90 days old at the start of the experiment. The rats were individually housed in wire mesh cages with food and water available ad lib. The lights in the animal colony came on at $0700 \mathrm{~h}$ and terminated at $1900 \mathrm{~h}$.

Apparatus. The apparatus used is described in detail elsewhere (Ross \& Randich, 1984). Briefly, two identical circular copper plates mounted on Plexiglas stands, with clear Plexiglas cylinders fitted on top of each, were used. One copper plate, designated the shock plate, was connected to a high-voltage shock source at the base of the plate and by a stainless steel wound clip implanted in the animals' back, allowing for presentation of a 1-sec, 2-mA shock US. The second copper plate, designated the hot plate, was contained within a thermostatically controlled water bath, which maintained a constant surface temperature of $51^{\circ} \pm 0.5^{\circ} \mathrm{C}$ across the plate. The hot plate has been used to index pain sensitivity and is thought to sample highly integrated escape responses (Hayes et al., 1978). Decreased sensitivity to pain (analgesia) is inferred from increased response latencies relative to the latencies of appropriate controls. Identical $150-\mathrm{W}$ projector lamps were mounted on the Plexiglas stand of each copper plate, $4.5 \mathrm{~cm}$ above the surface and $3 \mathrm{~cm}$ outside the Plexiglas cylinders. These lamps provided the visual CS.

Behavioral observation procedures. Systematic observations of each rat's behavior while on the shock plate were made during the $30-\mathrm{sec}$ visual CS presentation or during the corresponding interval for rats in groups that did not receive the visual CS. For each rat, a single behavior was scored every $5 \mathrm{sec}$, beginning $5 \mathrm{sec}$ following CS onset or the corresponding temporal interval. Thus, each rat's behavior was observed and scored six times during the 30 -sec 
interval. Individual behaviors were converted to percentages of total behavior to facilitate between-experiment comparisons.

Two major categories of behavior were recorded. Freezing behavior was the primary category of interest, and was defined as the absence of movement except for that resulting from respiration. Acquisition of freezing behavior was used to index an acquired emotional reaction, that is, fear (Bouton \& Bolles, 1980; Fanselow, 1980). The second category of behavior was general activity, which consisted of the following subcategories: (1) rearing-standing on hind legs with the front paws above the plate surface, (2) jumpingall four paws above the surface of the plate, (3) walking-gross body movements of legs and head, and (4) grooming-licking the paws, face, or body. These behaviors were collapsed into a general activity measure to facilitate presentation and because individually they did not vary systematically with conditioning treatments.

Procedure. Each rat was anesthetized with ether. The hair was removed from the dorsal surface of the back and a 9-mm stainless steel wound clip was implanted approximately $1.5 \mathrm{~cm}$ caudal and on the midline of the shoulder area. The rats then received a 24-h postsurgical recovery period.

The rats were assigned randomly to one of five treatment conditions ( $\mathrm{N}=16 /$ group), and the conditioning phase was started. All groups received a single 2 -min trial on each of 5 consecutive days. First, a shock lead was attached to the implanted wound clip while the rat was in a holding cage. The rat was then placed in the center of the shock plate, and the trial was initiated. At the end of the trial, the rat was removed and returned to the holding cage. Thus, the rats differed only with respect to the type of stimuli and temporal arrangement of the stimuli presented during this 2-min period. These treatments are shown in Figure 1.

\section{PROCEDURES}

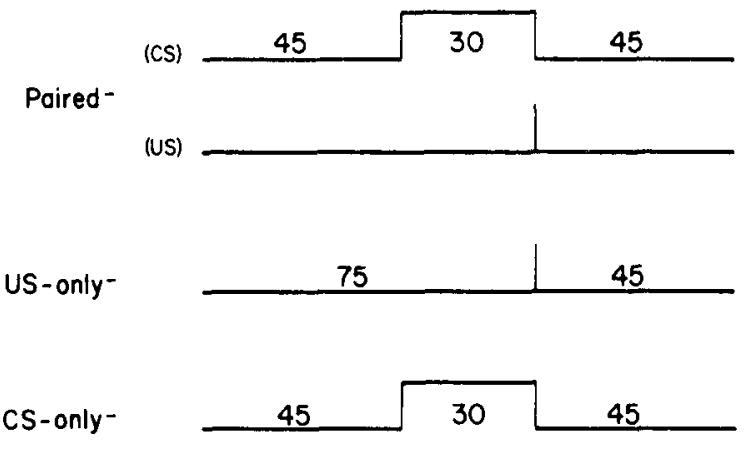

Apporatus -

120

(F)

30 90

Unpoired-

(B)

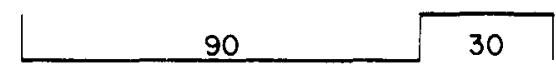

TIME IN SECONOS

Figure 1. Temporal arrangements of stimulus events for the various groups of Experiment 1. Note that for the unpaired group, two trial types are shown $(F=$ forward and $B=$ backward $)$.
Rats in the paired condition received a 45 -sec pre-CS interval, followed by a $30-\mathrm{sec}$ light CS, which terminated with a $1-\mathrm{sec}, 2-$ $\mathrm{mA}$ shock US, and a 45-sec post-CS interval, which terminated with removal of the rat from the plate. Rats in the US-only condition received a 75 -sec pre-CS interval, presentation of the shock US, and a 45-sec post-CS interval. Rats in the CS-only condition received a 45 -sec pre-CS interval, followed by presentation of the 30 -sec light CS and then a $45-\mathrm{sec}$ post-CS interval. Note that in the USonly and CS-only conditions the appropriate CS or US event was presented at the temporal location corresponding to that of the paired condition. Rats in the apparatus-only condition received no stimulus presentations during the 2 -min period on the plate. Finally, rats in the explicitly unpaired condition received two different types of trials. These rats received three "forward" trials during which the light CS was presented upon placement of the rat onto the plate; this was followed by a $90-\mathrm{sec}$ interval, and then by a 1 -sec shock and removal of the rat from the plate. These animals also received two "backward" trials, which consisted of a 1 -sec shock presentation upon placement of the rat on the plate, a 90 -sec interval, and a 30 -sec light CS, which terminated with the rat's removal from the plate. The order of presentation of these two types of trials was randomized over the 5 conditioning days, with the restriction that no more than two of the same trial type could occur consecutively.

On the following day, a single test session was conducted. Half the rats in each group were assigned to each of two testing procedures. This allowed for 10 independent groups ( $N=8$ /group) designated according to conditioning treatment and testing procedure. In the CS-test procedure, all rats received a 45 -sec exposure to the shock plate with no CS present, followed by transfer to the hot plate, where the visual CS was present. The context-test procedure was identical except that the visual CS was never presented during testing. In both test procedures, the latency in seconds to either lick a hind paw or jump from the plate surface was used as a behavioral index of pain sensitivity. However, since the jump response on the hot plate never accounted for more than $5 \%$ of any groups' behavior, all behaviors were categorized as paw licks. An upper limit of $120 \mathrm{sec}$ to respond was also imposed to prevent tissue damage. All statistical comparisons were conducted with $\mathrm{p} \leq .05$.

\section{Results and Discussion}

The mean percentage of freezing behavior in each of the five different conditioning treatment groups across the conditioning phase of Experiment 1 are shown in Figure 2.

Freezing behavior was acquired rapidly in both the paired and US-only conditions. Although acquisition of freezing also occurred in the unpaired condition, it did not attain the levels shown by the paired and US-only groups by the end of conditioning. In the unpaired condition, freezing was maintained at moderate levels over the final three sessions. Freezing remained at low and relatively stable levels in the CS-only and apparatus-only groups. These observations are supported by the results of the repeated measures analysis of variance, which showed significant effects for groups $[F(4,75)=36.0]$ and days $[F(4.300)=53.0]$ and a significant groups $\times$ days interaction $[F(16,300)=8.37]$. Individual group comparisons of freezing on Days 2 and 5 were conducted using the Tukey multiple comparison procedure. On Day 2. the paired, US-only, and unpaired groups did not differ, but each of these groups differed significantly from the CS-only and apparatus-only groups, which did not differ from each other. On Day 5 , the unpaired group 


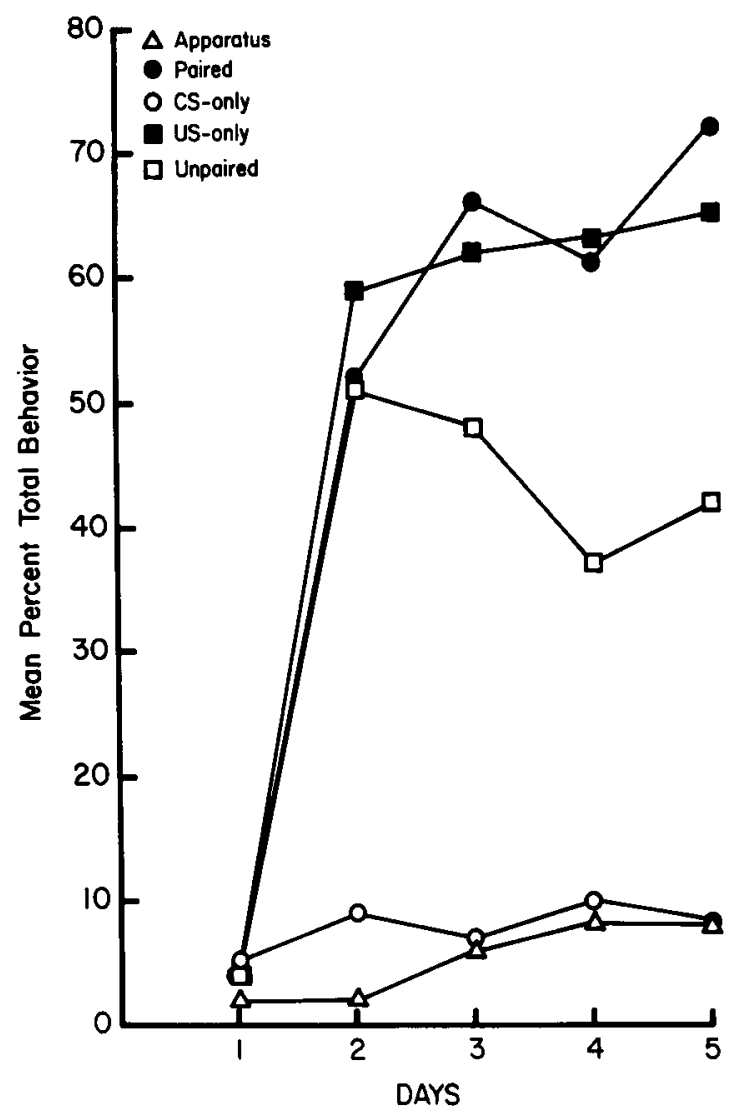

Figure 2. Mean percentage of freezing behavior for the various groups of Experiment 1 during the conditioning phase.

showed significantly less freezing than either the paired or the US-only group, and all of these groups showed significantly greater freezing than either the CS-only or apparatus-only group. Moreover, the paired and US-only groups did not differ, and the CS-only and apparatus-only groups did not differ.
The mean latency to paw lick and corresponding standard error of the mean for each of the 10 groups during the testing procedure are shown in Figure 3. Note that the filled bars (left) indicate groups tested in the presence of the CS and the open bars (right) indicate groups tested in the absence of the CS within each of the five different conditioning treatments.

Rats in the paired group that received the CS-test procedure showed substantially longer response latencies than rats in any other group, regardless of the test procedure. Furthermore, whether the test occurred in the presence or in the absence of the CS, there was a tendency for rats in the groups that received shock presentations during the conditioning phase (the paired, US-only, and unpaired groups) to show somewhat longer response latencies than rats in groups not receiving the shock US (the CS-only and apparatus-only groups). This finding suggests that some conditioning of contextual stimuli and/or handling cues may have occurred in groups receiving the shock US.

These findings were only partially supported by the results of a two-way analysis of variance on response latencies during the test session. This analysis revealed significant effects for conditioning treatment $[\mathrm{F}(4,70)=$ 6.77] and test procedure $[F(2,70)=4.76]$, but no significant conditioning treatment $\times$ test procedure interaction. Individual group comparisons using the Tukey procedure indicated that rats that received the paired conditioning treatment and the CS-test procedure had significantly longer response latencies than all other groups. All other comparisons were nonsignificant, indicating that the apparent increases in hot-plate response latencies for the remaining groups that had received the shock US during the conditioning phase (e.g., the US-only/context-test group) were not statistically significant.

These results indicate that the analgesia manifested by rats that received the paired conditioning teatment and the CS-test procedure was a consequence of associative learn-

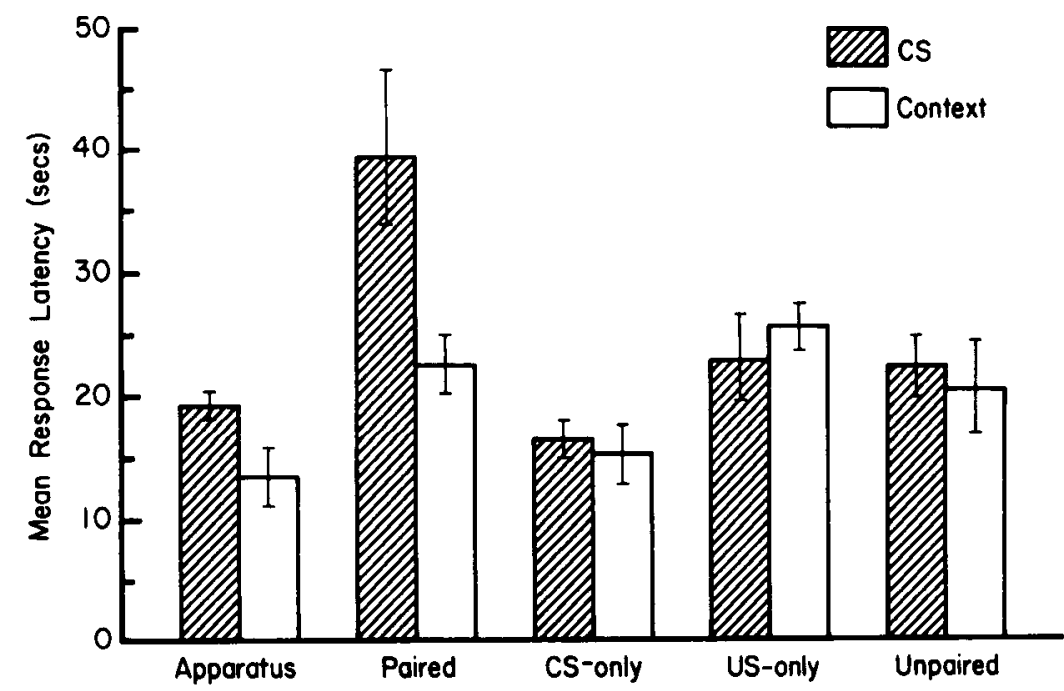

Figure 3. Mean response latency and corresponding standard error for all groups during the test session of Experiment 1. Filled bars (left) indicate the CS-test procedure, and open bars (right) indicate the context-test procedure within each condition. 
ing processes. The behavior of rats in a variety of Pavlovian control procedures, including explicitly unpaired CS and US, suggests that nonassociative factors are not contributing to the observed response. Moreover, rats in the paired treatment showed much longer latencies when tested in the presence of the CS than when tested in its absence, indicating that responding was clearly under control of the CS and that contextual stimuli contributed virtually nothing to the analgesia observed in this experimental situation.

The lack of correspondence between the acquisition of freezing behavior and increased response latencies on the hot plate is relevant to an alternative explanation of our results. For instance, it could be argued that the increased response latencies in the paired condition relative to those among the controls are not the result of reduced sensitivity to pain, but are due to the transfer of a response acquired during conditioning that is incompatible with the paw-lick response (cf. Blair, Galina, Holmes, \& Amit, 1982). In particular, perhaps freezing behavior in the paired group transferred to the hot plate and interfered with performance of the paw-lick response. However, the transfer of an incompatible response seems unlikely. Rats in the US-only condition showed the same amount of freezing behavior as did rats in the paired condition and would be expected to show equivalent transfer of freezing to the test situation, but rats in the US-only group failed to manifest analgesia.

\section{EXPERIMENT 2}

Experiment 2 was conducted to determine the number of discrete visual CS-US pairings required for the acquisition of conditioned analgesia using the present conditioning and analgesiometric assay procedures. Independent groups of rats received a total of $1,2,5$, or 10 pairings of a visual CS with a shock US. For all groups, a single conditioning trial was presented on each day and testing for analgesia occurred $24 \mathrm{~h}$ after the final conditioning session. Control groups, which received an equivalent amount of exposure to CS and US events in an explicitly unpaired manner, were included for comparisons in each experimental condition.

\section{Method}

Subjects and Apparatus. Sixty-four experimentally naive Sprague-Dawley rats served as subjects. The apparatus, behavioral observation procedure, running protocol, and subject factors were as in Experiment 1.

Procedure. All rats received the wound-clip implant as described previously. Twenty-four hours later, the rats were assigned randomly to one of eight groups ( $\mathrm{N}=8$ /group) and conditioning was begun.

Rats in the four paired groups received a single 2-min trial each day for a total of $1,2,5$, or 10 consecutive days; the running protocol and temporal parameters were identical to those described for the paired group of Experiment 1 (see Figure 1). Rats in the four unpaired control groups received the identical number of stimulus presentations over an equal number of days; the protocol was identical to that described for the explicitly unpaired condition of Ex- periment 1 (see Figure 1). However, the varying number of trials prevented full use of the unpaired procedure. Rats in the unpaired 1-day group received a single long-delay "forward" trial, as shown in Figure 1; rats in the unpaired 2-day group received a "forward" and "backward" trial; rats in the unpaired 5-day group received three "forward" and two "backward" trials in random order; and rats in the unpaired 10-day group received five "forward" and five "backward" trials in a random order. In the conditioning phase of the experiment, behavioral observations were made during the 30-sec visual CS in all eight groups according to the procedures described previously.

Twenty-four hours after the final conditioning session for each paired group and matched unpaired group, testing for conditioned analgesia was conducted in the manner of the CS-test procedure described for Experiment 1. Specifically, the rats received a 45sec exposure to the shock plate with no CS present, followed by transfer to the hot plate with the visual CS present. The latency to lick a hind paw or to jump was recorded for all groups.

\section{Results and Discussion}

The mean percentage of freezing behavior during the visual CS for each paired experimental group and each unpaired control group is shown in Figure 4. Consecutive panels show behavior for the 1-, 2-, 5-, and 10-day groups, respectively.

Paired and unpaired groups that received a single conditioning trial showed minimal freezing during the CS presentation. Groups that received two conditioning trials showed acquisition of freezing by the second trial, but although freezing remained at substantial levels in the 5and 10-day paired groups, freezing declined in the matched unpaired groups over the remaining conditioning trials.

These observations were supported by separate analyses of variance which contrasted each paired group with its matched unpaired control within the four treatment levels. The paired 1-day group did not significantly differ from the unpaired group. An analysis of the 2-day groups revealed a significant effect for days $[F(1,14)=40.57]$, but no effect for either groups or the groups $\times$ days interaction. An analysis of the 5-day groups revealed a significant effect of days $[F(4,56)=26.00]$ and a significant days $\times$ groups interaction $[F(4,56)=6.92]$. In the 10-day groups, the analysis revealed a significant effect of group $[F(1,14)=8.20]$ and days $[F(99,126)=5.56]$, but no interaction between groups and days. Collectively, these results indicate that significant acquisition of freezing behavior is seen during the second conditioning trial in both paired and unpaired groups, and that with continued conditioning trials freezing is significantly greater in the paired than in the unpaired treatment.

The mean latency to respond in the hot-plate assay and standard error of the mean for each group are shown in Figure 5. In general, rats in the 2-, 5-, and 10-day paired groups showed longer hot-plate latencies than their matched unpaired controls, whereas rats in both the paired and the unpaired 1-day groups showed virtually identical response latencies that were similar to those of the 2-, 5-, and 10-day unpaired groups. The results of a two-way analysis of variance partially supported these observations. 


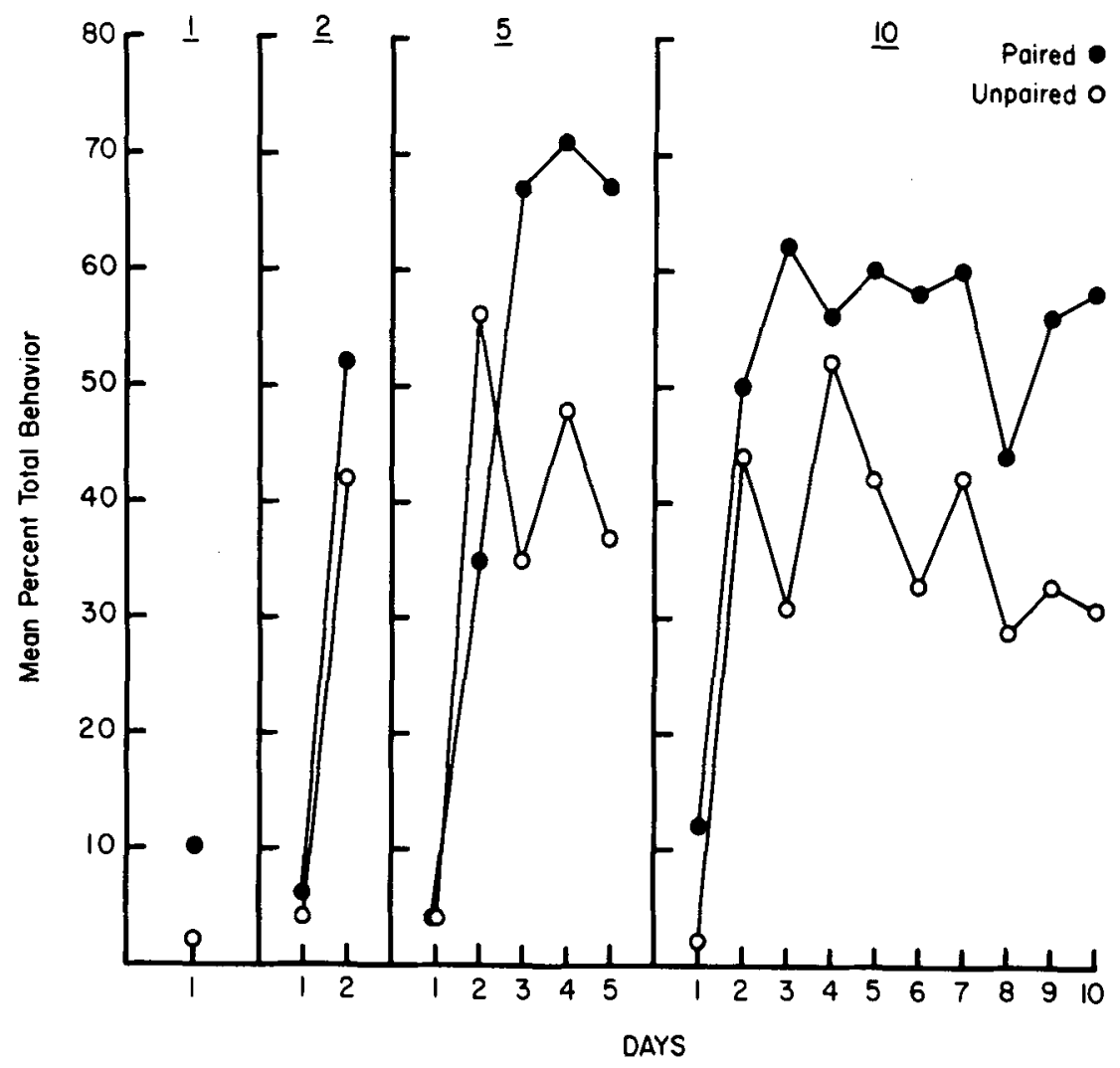

Figure 4. Mean percentage of freezing behavior of the paired (filled) and matched unpaired (open) control groups at each level of training in Experiment 2.

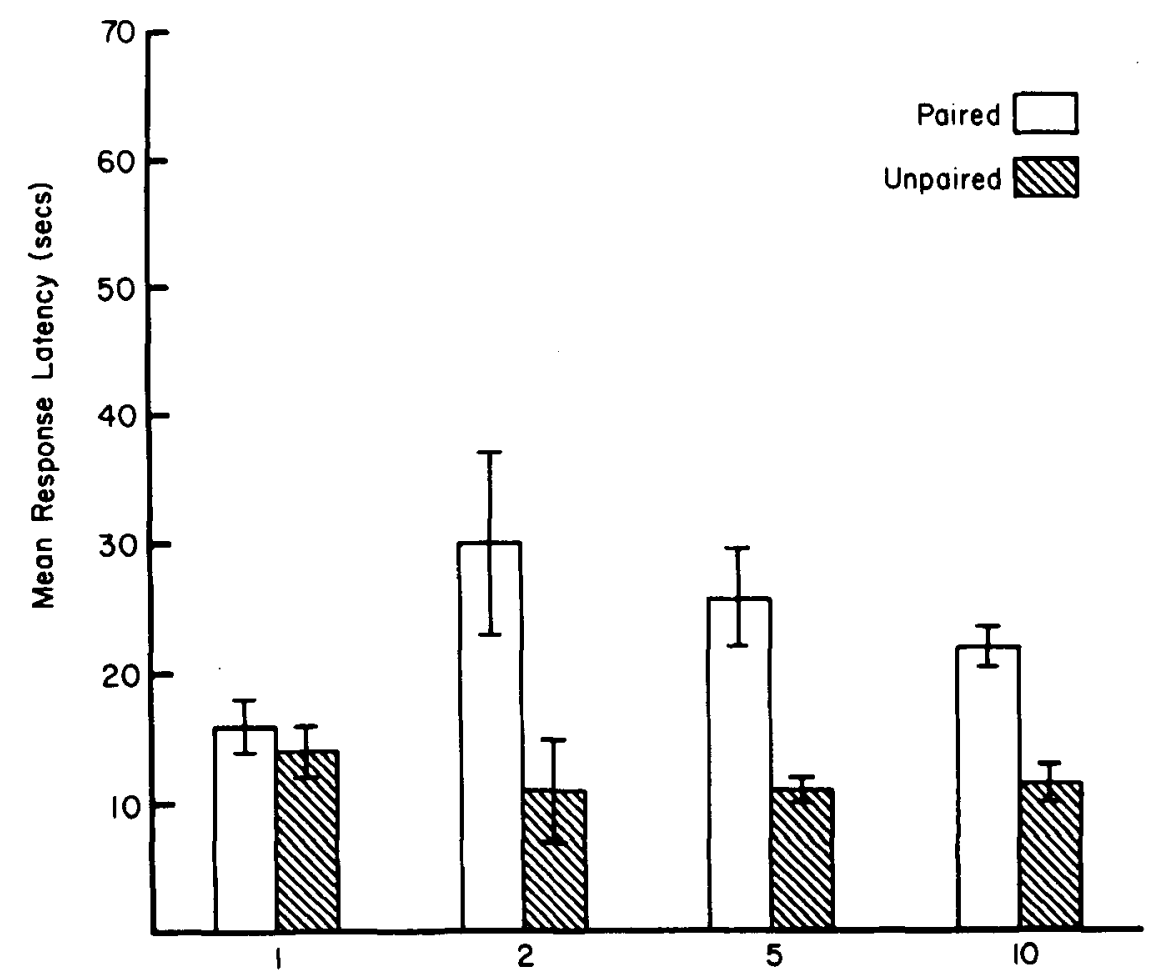

Figure 5. Mean response latency and corresponding standard error for the paired (open) and unpaired (filled) control groups during the test session of Experiment 2. 
This analysis revealed a significant effect for paired versus unpaired conditioning $[\mathrm{F}(1,56)=27.24]$ and a significant interaction of trial type and number of trials $[F(3,56)=2.21]$, but no effect of number of trials. Individual group comparisons, using the Tukey procedure, revealed significant differences between the 2-, 5-, and 10-day paired groups and their matched unpaired controls, but no significant difference between the 1-day paired and unpaired groups.

In the present experiment, we obtained no evidence of conditioned analgesia in the groups that received a single conditioning trial. All groups that received two or more paired conditioning trials manifested conditioned analgesia, but increasing the number of CS-US pairings beyond two did not significantly increase the level of analgesia. Indeed, there was a nonsignificant trend for a reduction in conditioned analgesia with increased CS-US pairings. Thus, in the present experimental situation, two pairings of the visual CS and shock US are sufficient for the acquisition of conditioned analgesia.

\section{EXPERIMENT 3}

Experiment 3 examined the effects of an extinction procedure on performance of previously established conditioned analgesia. Two groups of rats received five CSUS pairings during a conditioning phase, and then either 5 or 10 extinction trials in which the CS was presented alone. Two other groups of rats received the identical conditioning treatment but remained in their home cages for a period of time equal to the duration of the extinction treatment ( 5 or 10 days). Finally, two groups of rats received 5 days of an explicitly unpaired procedure during conditioning, and then remained in their home cages for either 5 or 10 days. All groups then received a single test of pain sensitivity following either the final extinction session or the appropriate retention interval.

\footnotetext{
Method

Subjects. Forty-eight experimentally naive male Sprague-Dawley rats served as subjects. The apparatus, behavioral observation procedure, basic running protocol and subject factors were as described for the previous experiments.

Procedure. All rats received the wound-clip implant and the 24-h recovery period. The rats were assigned randomly to one of two treatment conditions, and the conditioning phase of the experiment was begun.

Rats in the paired condition $(n=32)$ received a single 2-min trial each day for a total of 5 days, according to the identical experimental protocol and temporal parameters used for the paired groups in the previous experiments (see Figure 1). Rats in the unpaired condition $(n=16)$ received the identical experimental protocol reported for the 5-day explicitly unpaired groups of the preceding experiments. During the conditioning phase, behavioral observations were made during each $30-\mathrm{sec}$ light CS in all groups.

On the day following the final conditioning session, rats in the paired treatment were assigned randomly to one of four independent groups ( $N=8 /$ group), and rats in the unpaired condition were assigned randomly to one of two groups ( $\mathrm{N}=8 /$ group). Two groups
}

of rats that received CS-US pairings received an extinction procedure in which the visual CS was presented in the absence of the shock US. Group E5 received a single trial for each of 5 consecutive daily sessions, and Group E10 received 10 such extinction sessions. The remaining paired groups received either a 5-day (Group HC5) or a 10-day (Group HC10) retention interval in the home cage. The two unpaired groups also remained in the home cage for either 5 (Group UNP5) or 10 days (Group UNP10).

Twenty-four hours after the final extinction session or the appropriate retention interval, all groups received a single test session for pain sensitivity. Testing was as previously described for the CS-test condition.

\section{Results and Discussion}

The mean percentage of freezing behavior during the CS for both the paired and unpaired groups during the conditioning phase is shown in the left panel of Figure 6. The right panel of Figure 6 presents freezing behavior for the two groups that received extinction trials for either 5 or 10 sessions.

As in the preceding experiments, rats in the paired and unpaired conditions showed rapid acquisition of freezing behavior, followed by a decline in freezing behavior to moderate levels in the unpaired groups and maintenance of freezing in the paired groups. This observation was supported by an analysis of variance that showed a significant effect for paired versus unpaired conditioning $[F(4,184)=8.37]$ and a significant trial type $\times$ days interaction $[\mathrm{F}(4,184)=5.22]$.

Group E5 did not evidence a significant decline in freezing behavior during the CS presentation over the course of extinction, but a reliable decrement in freezing behavior was obtained in Group E10, particularly following Day 6 of extinction. These observations were supported by separate randomized blocks analyses of variance, which revealed a significant days effect only in Group E10 $[F(9,63)=5.65]$. In addition, separate analyses of variance showed no significant differences in the freezing behavor of Groups E5 and E10 across Sessions 1-5 of the extinction phase or during Session 5 only. Thus, the extinction treatment was effective in significantly reducing freezing behavior only in Group E10.

The mean latency to respond to thermal stimulation and the corresponding standard error of the mean for each group are shown in Figure 7.

Group HC5 (5-day retention control) showed longer latencies to respond than either Group E5 (5-day extinction group) or Group UNP5 (explicitly unpaired retention control). An analysis of variance indicated a significant between-groups effect $[\mathrm{F}(2,21)=7.91]$. Individual group comparisons revealed significantly longer latencies in Group HC5 than in either Group E5 or Group UNP5, which did not differ.

Similar results were obtained in the 10-day groups. An analysis of variance indicated a significant between-groups effect $[F(2,21)=5.94]$ for the 10-day treatments. Group HC10 had significantly longer response latencies than Group E5, but Group HC10 did not differ from 


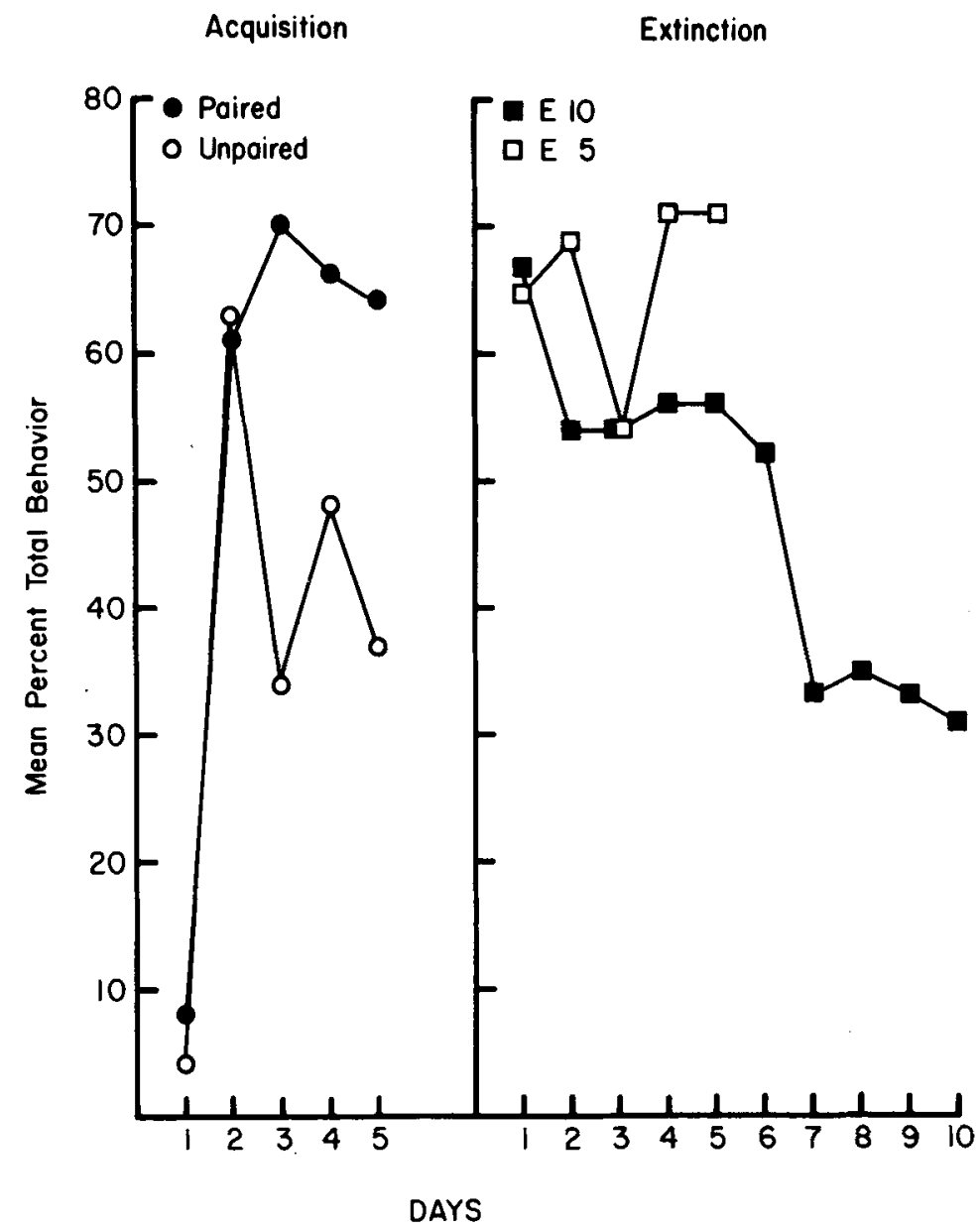

Figure 6. Mean percentage of freezing behavior for the paired and unpaired conditions during acquisition (left panel) and for the 5- and 10-day groups during extinction (right panel) of Experiment 3.

Group UNP10. Finally, two additional post hoc comparisons revealed that Groups HC5 and HC10 did not differ and that Groups E5 and E10 did not differ.

The results of Experiment 3 indicate that five extinction trials do not reduce freezing behavior, but are sufficient to eliminate conditioned analgesia (Group E5) as compared with either retention controls (Group HC5) or explicitly unpaired retention controls (Group UNP5). Additional extinction trials were effective in reducing freezing behavior (Group E10). Thus, within the present procedures conditioned analgesia can be decreased by using a standard extinction procedure.

\section{EXPERIMENT 4}

Experiment 4 was a preliminary attempt to examine whether the administration of the opiate receptor antagonist naloxone would attentuate or eliminate conditioned analgesia evoked by a visual CS previously paired with a shock US. Previous work that addressed unconditioned shock-induced analgesia using these procedures (Ross \& Randich, 1984) had revealed no effect of nalox- one pretreatment on the unconditioned response. Reversal of analgesia by an opiate receptor blocker provides some indication of possible mediation by an endogenous opioid pain-inhibition system (cf. Drugan et al., 1982; Fanselow \& Baackes, 1982). Two groups of rats received initial training with either paired or unpaired stimulus presentations. Prior to a test of pain sensitivity, half the rats in each condition were injected with naloxone and the remaining rats were injected with saline vehicle.

\section{Method}

Subjects and Apparatus. Thirty-two experimentally naive Sprague-Dawley rats served as subjects. The apparatus, behavioral observation procedure, running protocol, and subject factors were as described for the previous experiments.

Procedure. All rats received implantation of a wound clip and the 24-h recovery period. The rats were then divided into two groups $(\mathrm{N}=16 /$ group), and the conditioning phase of the experiment was begun.

Rats in the paired condition received a single CS-US pairing on each of 5 consecutive days, as described for the paired groups in the previous experiments. Rats in the unpaired group received an identical number of stimulus presentations, but these were distributed according to the protocol described previously for the explicitly un- 


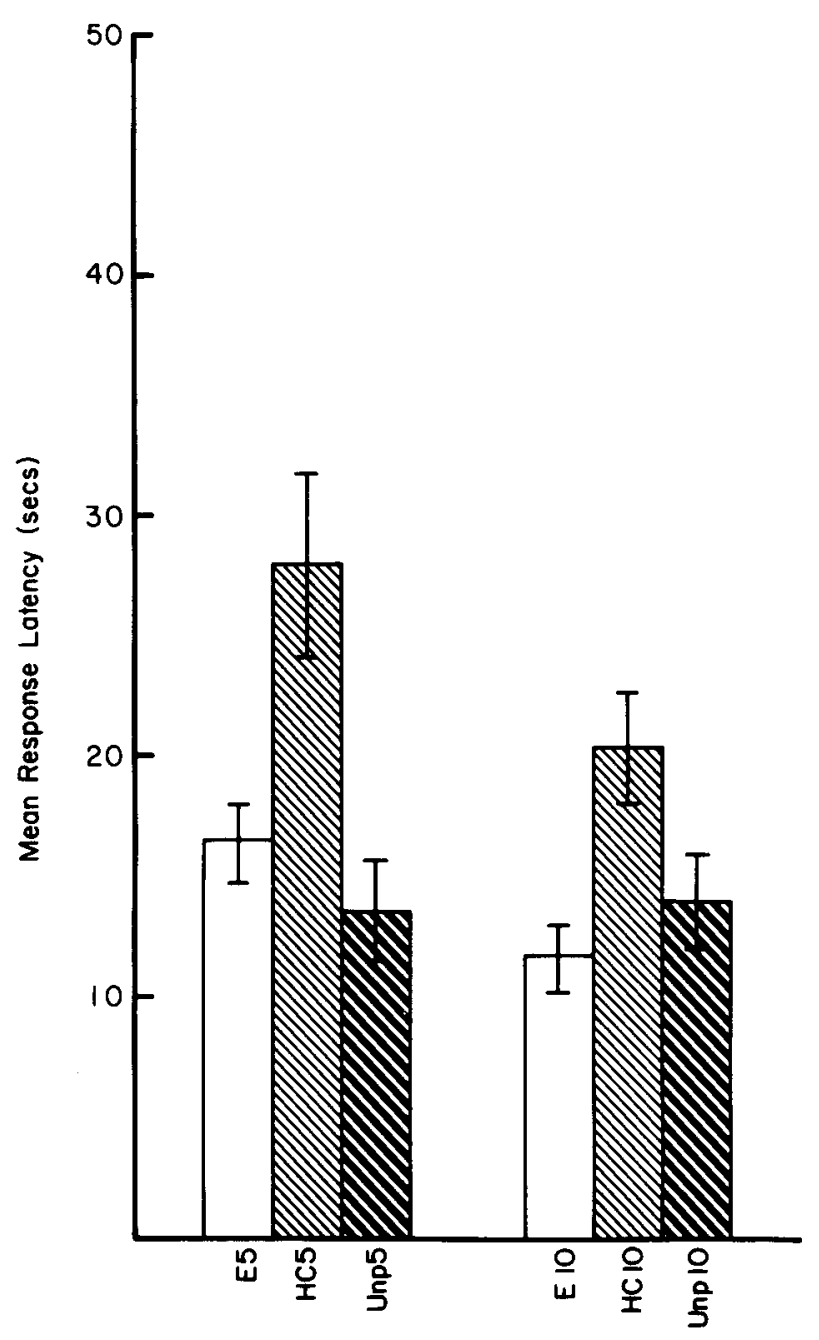

Figure 7. Mean response latency and corresponding standard error for all groups during the test session of Experiment 3 .

paired procedure. During the condition phase, behavioral observations were made during the 30 -sec light $\mathrm{CS}$ in both groups.

On the day following the last conditioning trial, half the rats in the paired condition (Group Paired Naloxone) received an intraperitoneal injection of naloxone $\mathrm{HCl}$ at $10 \mathrm{mg} / \mathrm{kg}$ of ad-lib body weight $10 \mathrm{~min}$ prior to the start of the test session and the remaining rats in the paired condition (Group Paired Saline) received an injection of an equal volume of saline vehicle. Similarly, half the rats in the unpaired condition (Group Unpaired Naloxone) received $10 \mathrm{mg} / \mathrm{kg}$ of naloxone and the remaining rats in this condition (Group Unpaired Saline) received an equivalent volume of saline vehicle.

All rats then received the CS-test procedure described previously. The latency to lick the hind paw or jump was recorded.

\section{Results and Discussion}

The mean percentage of freezing behavior during the $\mathrm{CS}$ for both the paired and unpaired conditions during the conditioning phase of the experiment are shown in Figure 8.

Rats in the paired and unpaired groups showed rapid acquisition of conditioned freezing. The percentage of freezing behavior continued to increase in the paired group, while moderate levels of freezing occurred in the unpaired group over Days 3-5. An analysis of variance indicated significant effects of paired versus unpaired conditioning $[F(1,30)=6.56]$ and days $[F(4,120)=32.69]$, but no reliable interaction. These outcomes are similar to those obtained in the previous experiments, showing significantly greater freezing behavior in the paired than in the unpaired conditions.

The mean latency to respond in the hot-plate assay and corresponding standard error for each group are shown in Figure 9.

Rats in the groups that had received paired trials during conditioning showed substantially longer response latencies than the rats that had received unpaired trials. Importantly, naloxone administation appears to have had no effect in any group. These views were supported by an analysis of variance showing a significant effect for paired versus unpaired conditioning $[F(1,28)=13.07]$ but no significant effect for either naloxone versus saline treatment or the interaction between those factors. Thus, conditioned analgesia manifested by rats in the paired condition was unaffected by prior administration of the opiate receptor antagonist naloxone.

In our previous work that examined unconditioned analgesia evoked by the same shock US (Ross \& Randich, 1984), intraperitoneal administration of $1,5,10$, or $20 \mathrm{mg} / \mathrm{kg}$ of naloxone was also ineffective in attenu-

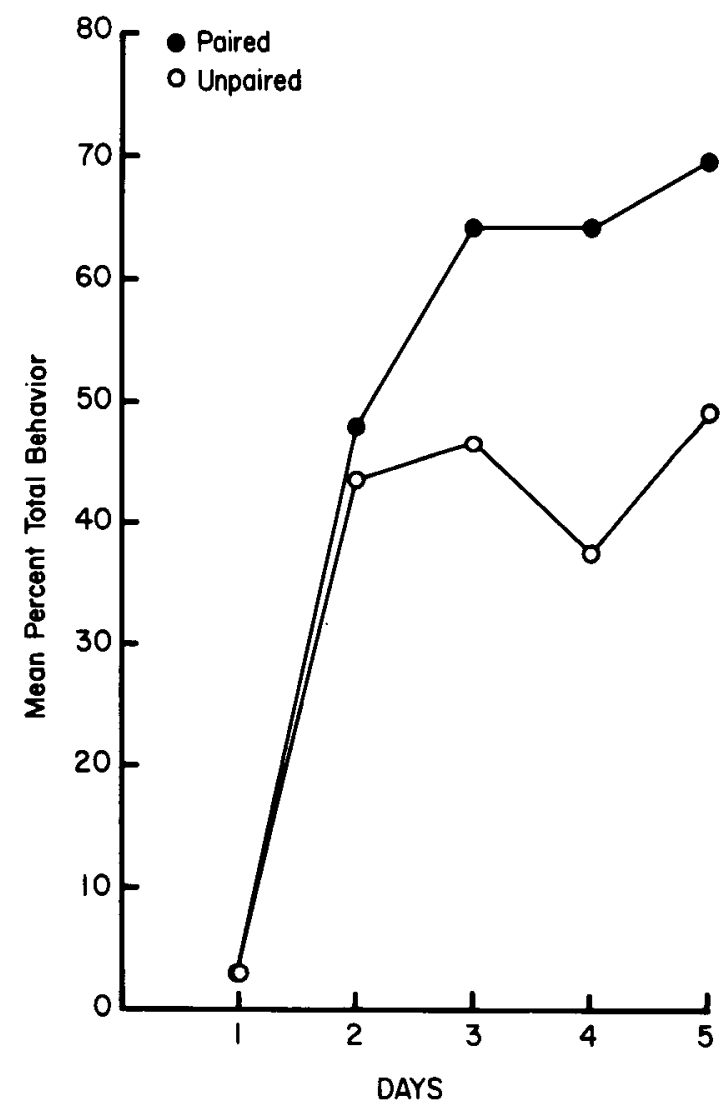

Figure 8. Mean percentage of freezing behavior for the paired and unpaired groups of Experiment 4 during the conditioning phase. 


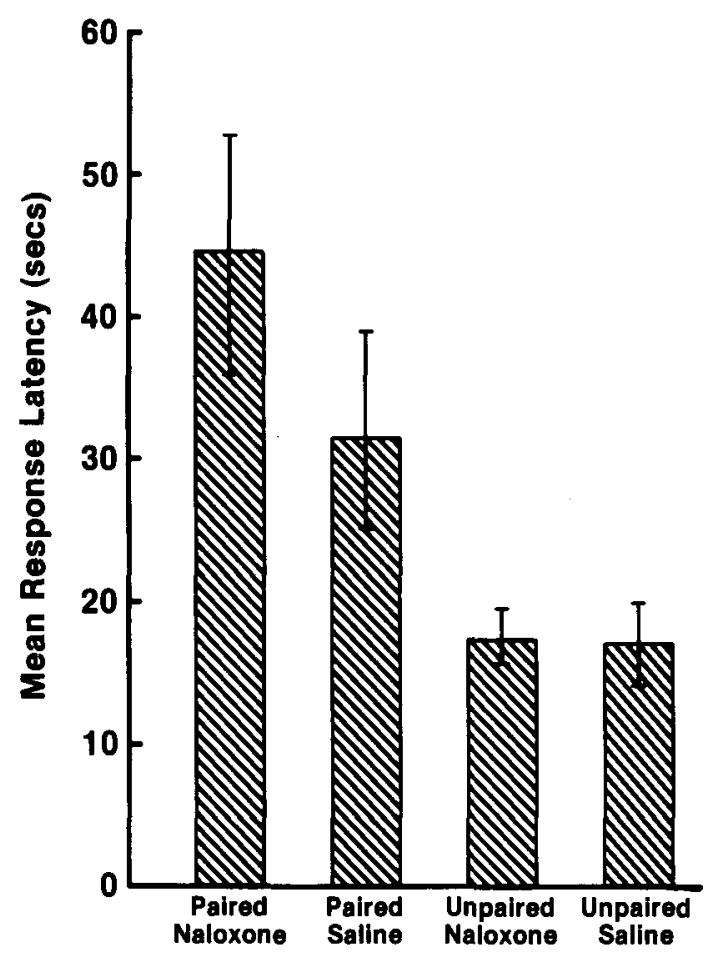

Figure 9. Mean response latency and corresponding standard error for the four groups during the test session of Experiment 4.

ating the analgesic response. The results of both that experiment and the present Experiment 4 suggest that this form of analgesia may be mediated by an endogenous pain-inhibition system in which opioids play no major role-that is, a nonopioid system (Drugan et al., 1982; Grau, Hyson, Maier, Madden, \& Barchas, 1981; Lewis, Cannon, \& Liebeskind, 1980; Lewis, Sherman, \& Liebeskind, 1981; Spiaggia et al., 1979). However, these null outcomes do not preclude a role for endogenous opioids in mediating both the unconditioned and the conditioned analgesia observed using the present procedures. Indeed, recent evidence (Panerai, Martini, Sacerdote, \& Mantegazza, 1984) indicates that opioid and nonopioid analgesia may be mediated by different opioid receptors, naloxone having a low affinity for opioid receptors that mediate "nonopioid" analgesia.

\section{GENERAL DISCUSSION}

The present series of experiments systematically investigated the acquisition of both conditioned analgesia (indexed by increased latencies to respond in the hot-plate assay of pain sensitivity) and conditioned fear (indexed by increased freezing behavior) to a visual CS paired with a shock US. Experiment 1 established the associative basis of conditioned analgesia by showing significant increases in the latency to lick a hind paw in the presence of a visual CS previously paired with a shock US. Control groups receiving CS and US explicitly unpaired, CS only, US only, or apparatus only, showed no such increased latencies. Data were also obtained showing similar acquisi- tion of conditioned freezing behavior in the paired and US-only groups, and moderate acquisition of freezing in the unpaired group. Experiment 2 demonstrated that a minimum of two CS-US trials is required for acquisition of conditioned analgesia using the present experimental protocols. Experiment 3 demonstrated that conditioned analgesia can be extinguished by five CS-alone presentations. Extinction of conditioned freezing behavior was obtained following more extended nonreinforced presentations of the CS. Finally, in Experiment 4, we were unable to alter conditioned analgesia by prior administration of $10 \mathrm{mg} / \mathrm{kg}$ of the opiate receptor antagonist naloxone. These experiments provide convincing evidence that conditioned analgesia evoked by a discrete visual CS is established through associative learning rather than through some nonassociative process; in all four experiments, rats that received paired presentations of the visual CS and shock US showed significantly greater analgesia than rats that received explicitly unpaired presentations of the stimuli. These outcomes attest to the efficacy of the present protocols and make additional analyses of Pavlovian conditioning processes that mediate conditioned analgesia viable.

The use of both CS-test and context-test procedures in Experiment 1 also established that the conditioned analgesia shown in the paired condition was under primary control of the visual CS and was not evoked by contextual stimuli and/or handling cues also paired with the shock US. The failure to find conditioned analgesia to contextual stimuli in either the US-only or explicitly unpaired conditions, although consistent with our previous findings (Ross \& Randich, 1984, Experiments 1 and 2), is surprising given the outcomes of other studies (cf. Chance, 1980; Drugan et al., 1982; Fanselow \& Baackes, 1982; Hayes et al., 1978). Despite our efforts to minimize its influence, one explanation could be that generalization decrement was operating to mask analgesia conditioned to contextual stimuli in the US-only group of Experiment 1 (see also Ross \& Randich, 1984, Experiments 1 and 2) and perhaps in groups receiving explicitly unpaired training in each of the present studies. One potentially critical component of contextual stimuli provided by the apparatus used in these experiments, the copper plate surface, necessarily differed in temperature between conditioning (shock plate) and testing (hot plate) chambers. A generalization decrement account would hold that rats receiving US-only or explicitly unpaired trials on the shock plate should be responding primarily to contextual stimuli (e.g., the copper plate surface). Thus, responding might be substantially disrupted when rats are presented with a different contextual stimulus (i.e., the hot copper plate surface). In contrast, rats receiving pairings of the visual CS and shock US during conditioning should be responding primarily to the visual CS, which was identical in both chambers. Although some disruption of responding would be expected due to the change in contextual stimuli, suggesting that analgesia in our paired condition is somewhat underestimated, that effect 
should be less substantial than in the US-only and explicitly unpaired groups. The results of an unpublished experiment done in our laboratory are relevant to this generalization decrement account of our present data. Using the same experimental apparatus, testing procedure, and shock parameters, we presented independent groups of rats with 1,5 , or 10 contextual stimuli-US pairings, according to a protocol that differed from the present procedures used in the US-only group of Experiment 1 (see also Ross \& Randich, 1984, Experiments 1 and 2) only in that rats were removed from the shock plate upon US termination; no postshock interval was used. The conditions for the occurrence of generalization decrement in the unpublished study were identical to those described for the present experiments and for our previous work (Ross \& Randich, 1984). However, evidence for the acquisition of conditioned analgesia to contextual stimuli was obtained in both the 5- and 10-trial groups, relative to appropriate controls. These results suggest that a simple generalization decrement account of our failure to obtain evidence for conditioned analgesia using US-only and explicitly unpaired procedures is inadequate.

Certain aspects of the present experiments are also relevant to the perceptual-defensive-recuperative (PDR) model of fear and pain advanced by Bolles and Fanselow (1980), which currently offers the most complete account of how associative learning mediates conditioned analgesia. According to that model, presentation of the CS evokes an acquired expectancy of the US, which activates a central motivational state, specifically fear. This fear motivational state in turn engages both species-specific defensive behaviors (e.g., Bolles, 1970) and endogenous pain inhibition systems - that is, fear causes or produces analgesia (Bolles \& Fanselow, 1980; Fanselow \& Baackes, 1982; Fanselow \& Bo'les, 1979). Thus, a CS that elicits species-specific defensive behavior might also be expected to evoke analgesia, because both are indices of fear. In the present experiments, the PDR model would predict correspondence (a positive correlation) between freezing behavior, one of the rat's defensive behaviors used as an index of fear (Bouton \& Bolles, 1980; Fanselow, 1980), and hot-plate response latencies used as an index of pain sensitivity or analgesia. In general, the results of the present experiments are in agreement with predictions derived from Bolles and Fanselow's (1980) model. For example, in all of the present experiments, evidence for acquisition of conditioned analgesia to the visual CS was obtained only in rats that also displayed acquisition of freezing behavior to the CS during the conditioning phase (i.e., the paired groups). However, there are also a number of instances in which the occurrence of freezing behavior to the CS during conditioning, indicating acquisition of fear, did not predict the occurrence of conditioned analgesia during the test session. For example, in Experiment 1 , rats that received explicitly unpaired presentations of the visual CS and shock US acquired significant freezing behavior during the conditioning phase, relative to nonshocked controls. Despite the presence of condi- tioned fear (as indicated by freezing behavior), these rats failed to show conditioned analgesia (as indexed by increased response latencies on the hot plate). This outcome is easily accounted for by the PDR model by postulating a threshold that conditioned fear would need to exceed before endogenous pain inhibition systems are activated (cf. Fanselow, 1984). In the same study, rats that received US-only presentations displayed acquisition of freezing behavior equivalent to that seen in the paired group, but failed to show significant acquisition of conditioned analgesia during the test session. Any threshold level of fear required to activate endogenous pain inhibition systems should have been exceeded in this group. It could be argued that differences in conditioned fear between the paired and US-only groups of Experiment 1 may have been masked by ceiling effects, making inferences about equivalent levels of fear indexed by freezing behavior difficult. Although the presence of a ceiling effect in our freezing data is difficult to rule out entirely, note that both the paired and the US-only groups show a trend for increases in freezing behavior across Days 2-5 of Experiment 1 . Although this trend is not statistically significant, it suggests the possibility that both curves may still be rising at an equivalent rate, and therefore that no ceiling has been reached. In Experiment 3, rats that received five extinction trials showed no significant loss in freezing behavior, presumably indicating that substantial fear to the visual CS remained. However, these rats failed to show analgesia, as indexed by hot-plate response latencies. Although this outcome might not be anticipated by Bolles and Fanselow's (1980) model, this observed dissociation between freezing behavior and hot-plate response latencies could be due to these measures' being differentially sensitive indices of conditioned fear. In the absence of any independent evidence about the relative sensitivity of these indices, we cannot completely discount this possibility.

To further evaluate Bolles and Fanselow's (1980) assumption that a fear motivational state will produce analgesia, we used the occurrence of freezing behavior during the final conditioning session to predict analgesia during the hot-plate test session. We combined the data from the 8 rats in the paired/CS-test group of Experiment 1 with that of the 8 rats in the paired 5-day group of Experiment 2 and calculated a Spearman rank order correlation between freezing during Day 5 of conditioning and hot-plate response latencies during the subsequent test session. These groups were selected because they were treated identically and their data appeared to be consistent with predictions derived from the PDR model; both groups displayed substantial freezing behavior (indexing fear) and increased hot-plate response latencies (indexing analgesia). Although the PDR model would predict a positive correlation in this case, this analysis yielded a correlation coefficient of $-.47(\mathrm{~N}=16, \mathrm{p} \leq .05)$. The results of this analysis suggest that the occurrence of fear (as indexed by freezing behavior) could not be used to accurately predict the occurrence of analgesia (as indexed 
by hot-plate response latencies). Fanselow and Baackes (1982) have reported a negative within-cell correlation between freezing behavior and the occurrence of Formalininduced recuperative behavior (an index of pain sensitivity), which they interpreted as consistent with the PDR model. We believe that these different interpretations can be attributed to our use of a between-session correlation and Fanselow and Baackes's use of a within-session correlation. Fanselow and Baackes (1982) observed freezing and recuperative behavior concurrently, and it is reasonable to assume that if their rats were displaying one behavior (e.g., freezing) that would prevent display of alternative behaviors (e.g., recuperative behavior), thus resulting in an appropriate negative correlation. The negative correlation between freezing behavior and hot-plate response latencies which we have reported cannot be explained in a similar manner because we used a betweensessions procedure. However, it is worth noting an alternative, but related, explanation for our finding a negative correlation between freezing behavior and hot-plate response latencies. Fanselow (1984) has emphasized that such negative correlations might result from peripheral response competition between behaviors used to index pain sensitivity and other responses elicited by the stressor, in this case freezing. In the present case, this explanation would suggest that rats engaging in freezing behavior during the test session would experience increased contact time with the hot-plate surface and therefore show artificially decreased response latencies, relative to rats that did not engage in freezing. However, in Experiment 1, we provided evidence suggesting that transfer of a competing response (freezing) could not account for the longer hot-plate response latencies found in our paired groups. Recall that rats that received exposure to the shock alone (Group US-only) showed equivalent acquisition of freezing behavior during conditioning but failed to show acquisition of analgesia as indexed by hotplate response latencies. Furthermore, we calculated a Spearman correlation for these rats and also found a negative correlation between the final conditioning session and hot-plate response latencies during the test $(-.53, \mathrm{~N}=16$, $\mathrm{p} \leq .05$ ). It seems unlikely that a peripheral response account involving freezing behavior and increased contact time with the hot plate during testing offers an adequate account of our data.

The results of the present series of experiments suggest that a fear motivation state may not be a sufficient condition for activation of endogenous pain-inhibition systems as indicated by Bolles and Fanselow's (1980) PDR model. A recent study by Kinscheck, Watkins, and Mayer (1984) is also relevant to this issue. These investigators attenuated fear using either anatomical or pharmacological manipulations, but still observed acquisition of conditioned analgesia. Unfortunately, Kinscheck et al. (1984) did not provide a systematic analysis of behaviors indexing fear (such as freezing), but their results suggest that a fear motivational state may not be necessary for activa- tion of endogenous pain-inhibition systems. Currently, we favor examining an account that emphasizes the mediation of conditioned analgesia by an acquired expectancy or representation of the US. Such an account would have much in common with the assumptions of Bolles and Fanselow's PDR model, but would suggest that the expectancy or representation of the forthcoming US would directly activate pain-inhibition systems, rather than requiring the presence of a fear motivational state.

\section{REFERENCES}

Amir, S., Brown, Z. W., \& AMIt, Z. (1980). The role of endorphins in stress: Evidence and speculations. Neuroscience \& Biobehavioral Reviews, 4, 77-86.

Blair, R., Galina, Z. H., Holmes, L. J., \& Amit, Z. (1982). Stressinduced analgesia: A performance deficit or a change in pain responsiveness? Behavioral \& Neural Biology, 34, 152-158.

Bodnar, R. J., Kelly, D. D., Brutus, M., \& Glusman, M. (1980). Stress-induced analgesia: Neural and hormonal determinants. Neuroscience \& Biobehavioral Reviews, 4, 87-100.

Bolles, R. C. (1970). Species-specific defense reactions and avoidance learning. Psychological Review, 77, 32-48.

Bolles, R. C., \& FANSElow, M. S. (1980). A perceptual-defensiverecuperative model of fear and pain. Behavioral \& Brain Sciences, 3, 291-323.

Bolles, R. C., \& FANSELOW, M. S. (1982). Endorphins and behaviors. Annual Review of Psychology, 33, 87-101.

Bouton, M. E., \& Bolles, R. C. (1980). Conditioned fear assessed by freezing and by the suppression of three different baselines. Animal Learning \& Behavior, 8, 429-434.

Chance, W. T. (1980). Autoanalgesia: Opiate and non-opiate mechanisms. Neuroscience \& Biobehavioral Reviews, 4, 55-67.

Chance, W. T., \& Rosecrans, J. A. (1979a). Lack of cross-tolerance between morphine and autoanalgesia. Pharmacology, Biochemistry, \& Behavior, 11, 639-642.

Chance, W. T., \& Rosecrans, J. A. (1979b). Lack of effect of naloxone on autoanalgesia. Pharmacology, Biochemistry, \& Behavior, 22, 643-646.

Chance, W. T., White, A. C., Krynock, G. M., \& Rosecrans, J. A. (1979). Autoanalgesia: Acquisition, blockade, and relationship to opiate binding. European Journal of Pharmacology, 58, 461-468.

Drugan, R. C., Moye, T. B., \& Maier, S. F. (1982). Opioid and nonopioid forms of stress-induced analgesia: Some environmental determinants and characteristics. Behavioral \& Neural Biology, 35, 251-264

FANSELOW, M. S. (1980). Conditioned and unconditioned components of post-shock freezing in rats. Pavlovian Journal of Biological Sciences, 15, 177-182.

FANSELOW, M. S. (1984). Shock-induced analgesia on the formalin test: Effects of shock severity, naloxone, hypophysectomy, and associative variables. Behavioral Neuroscience, 98, 79-95.

Fanselow, M. S., \& BAACKES, M. P. (1982). Conditioned fear-induced opiate analgesia on the formalin test: Evidence for two motivational systems. Learning \& Motivation, 13, 200-221.

FansElow, M. S., \& Bolles, R. C. (1979). Triggering of the endorphin analgesic reaction by a cue previously associated with shock: Reversal by naloxone. Bulletin of the Psychonomic Society, 14, 88-90.

Grau, J. W., Hyson, r. L., Maier, S. F., Madden, J., \& Barchas. J. D. (1981). Long-term stress-induced analgesia and activation of the opiate system. Science, 213, 1409-1411.

Hayes, R. L., Bennett, G. J., Newlon, P. G., \& Mayer, D. J. (1978). Behavioral and physiological studies of non-narcotic analgesia in the rat elicited by certain environmental stimuli. Brain Research, 255 , $69-90$

Kinscheck, I. B., Watkins, L. R., \& MAYeR, D. J. (1984). Fear is not critical to classically conditioned analgesia: The effects of peria- 
queductal gray lesions and administration of chlordiazepoxide. Brain Research, 298, 33-44.

Lewis, J. W., Cannon, J. T., \& Liebeskind, J. C. (1980). Opioid and nonopioid mechanisms of stress analgesia. Science, 208, 623-625.

Lewis, J. W., Sherman, J. E., \& Liebeskind, J. C. (1981). Opioid and nonpioid stress analgesia: Assessments of tolerance and crosstolerance with morphine. Journal of Neuroscience, 1, 358-363.

Maclennan, A. J., Jackson, R. L., \& Maier, S. F. (1980). Conditioned analgesia in the rat. Bulletin of the Psychonomic Society, 15, 387-390.

Oliverio, A., \& Castellano, C. (1982). Classical conditioning of stress-induced analgesia. Physiology \& Behavior, 25, 171-172.

Panerai, A. E., Martini, A., Sacerdote, P., \& Mantegazza, P. (1984). X-Receptor antagonist reverse "non-opioid" stress induced analgesia. Brain Research, 304, 153-156.

Riley, A. L., Zellner, D. A., \& Duncan, H. J. (1980). The role of endorphins in animal learning and behavior. Neuroscience \& Biobehavioral Review's, 4, 69-76.
Ross, R. T., \& Randich, A. (1984). Unconditioned stress-induced analgesia following exposure to brief footshock. Journal of Experimental Psychology: Animal Behavior Processes, 10, 127-137.

Spiaggia, A., Bodnar, R. J., Kelly, D. D., \& Glusman, M. (1979). Opiate and non-opiate mechanisms of stress-induced analgesia: Cross tolerance between stressors. Pharmacology, Biochemistry, \& Behavior, 10, 761-765.

Watkins, L. R., Cobelli, D. A., \& MAYER, D. J. (1982). Classical conditioning of front paw and hind paw footshock induced analgesia (FSIA): Naloxone reversibility and descending pathways. Brain Research, 243, 110-132.

WATKINS, L. R., \& MAYER, D. J. (1982). Organization of endogenous opiate and nonopiate pain control systems. Science, 216, 1185-1192.

(Manuscript received December 15, 1984; revision accepted for publication May 22, 1985.) 